\title{
Interferometry Techniques with Heterogeneous VLBI Data
}

\author{
Tianyi Zhang ${ }^{1}$, Qiao Meng ${ }^{1+}$, Weimin Zheng ${ }^{2}$, Wei Liu ${ }^{1}$, Li Tong $^{2}$ and Quantao Yu ${ }^{1}$ \\ ${ }^{1}$ Southeast University, Nanjing, China \\ ${ }^{2}$ Shanghai Astronomical Observatory, Chinese Academy of Sciences, Shanghai, China
}

\begin{abstract}
Very-long-baseline interferometry (VLBI) is a powerful technique in radio astronomical observations. However, data recorded by different digital back ends in different observatories could be heterogeneous and are not able to be processed directly. This paper discusses a VLBI data transformer for heterogeneous data processing in the general situation. Furthermore, a direct-FX correlator architecture is proposed to perform correlation between heterogeneous data directly. Both method has been verified by computer simulations and quasar observations.
\end{abstract}

Keywords: VLBI, data transformer, correlator.

\section{Introduction}

Very Long Baseline Interferometry (VLBI) is a radio interferometry technique for precise position determination of radio sources on the sky [1]. It has become a significant approach in astronomy, geodesy, geodynamics and deep space exploration. Digital back ends (DBE) sample the analog signals and pack the radio data with essential information. Packed Data is then fed to correlators to perform cross-correlation and propagation delays between stations could be obtained from the phase of cross spectrum [2].

Normally, identical DBEs are usually used in VLBI observations which can give the package data in the same format. However, it still exists some situations where different DBEs would be used in the same observation, and they will produce heterogeneous data which the correlators could not process directly [3]. Firstly, the data are packed in different formats. Secondly, the samples would be recorded in different sampling types (real or complex) [4], [5]. Thirdly, the sample parameters of DBEs, including sample rate, baseband bandwidth and start frequency of baseband, would be different. Finally, there would be differences between the tuners before DBEs, therefore the baseband frequencies of each DBE may refer to different sky frequencies.

The most common solution for heterogeneous data processing is using data transformers to unify the sample parameters of VLBI data. Data in IFMS, VSR and VLBI Mk-5 formats are transformed to the common data format by different converters [6]. A software-based Hilbert transformer was used to translate between complex and real sampling raw data in international VLBI experiments of IKAROS [7]. However, these transformers only deal with the specific situation: a) the sample rate of complex data is half of that of real data so the baseband bandwidth are the same, b) the tuners before DBEs are configured to move the same sky frequencies to basebands.

This paper discusses the design of the data converter for heterogeneous data translation in general cases. Meanwhile, a direct FX correlation algorithm is proposed to make correlation between heterogeneous data directly. The rest of the correspondence is organized as follows. In section 2, the covert-before-correlation method is discussed and tested by computer simulation. Section 3 provides the direct FX correlation method and quasar experiment results. Conclusions are drawn in Section 4.

\footnotetext{
${ }^{+}$Corresponding author. Tel.: + 0086-25-83614269; fax: + 0086-25-83614269.
}

E-mail address: mengqiao@ seu.edu.cn. 


\section{Convert-before-correlation method for heterogeneous data}

The common solution is to convert heterogeneous data to an identical format so that designed correlators could perform correlation without modification. Our data converter deals with heterogeneous data with different sampling types and sample parameters.

The correct subband should be firstly chosen from original basebands for sky frequency alignment. The bandwidth of subband is limited by the overlapping bandwidth of the original basebands. There are two options to perform the alignment. In the first way, one data stream is the 'reference data' and the other one is converted to be the same. Another way is converted both data streams to the same type and sample rate. As the bandwidth of subband is usually smaller than the original bandwidth, the second way is chosen to reduce the computation of correlation.

Fig. 1 depicts the subband selection with the frequency domain representation during conversion. Real and complex data should be processed by different data converters, while Fig. 1 demonstrates all kinds of conversion together. The $f_{s 1}, f_{s 2}$ and $f_{s}$ are the sample rates of heterogeneous data and output. The sample rate of the output data is determined by its bandwidth and data type. The sample rate of real data should be larger than Nyquist frequency and it is twice than that of complex data with the same bandwidth. The subband selection is applied as a complex mixer. The frequency of the numerically controlled oscillator (NCO) should be configured as:

$$
f_{L O_{i}}=\left(f_{\text {start }_{i}}+\frac{B}{2}\right) / f_{s_{i}}, \quad i=1,2,
$$

where $f_{\text {start }}$ refers to the same sky frequency $f_{s k y}$ in its original baseband. Data types should be the same at the converter output, either real or complex. Real-to-complex conversion is performed by Hilbert transformers in [6] and [7], while it is performed with subband selection in the same time. An additional complex mixer should be applied after sample rate conversion to perform complex-to-real transform. Complex data after sample rate conversion is multiplied with $\exp (j \pi n / 2)$ and only the real part of its output is taken.

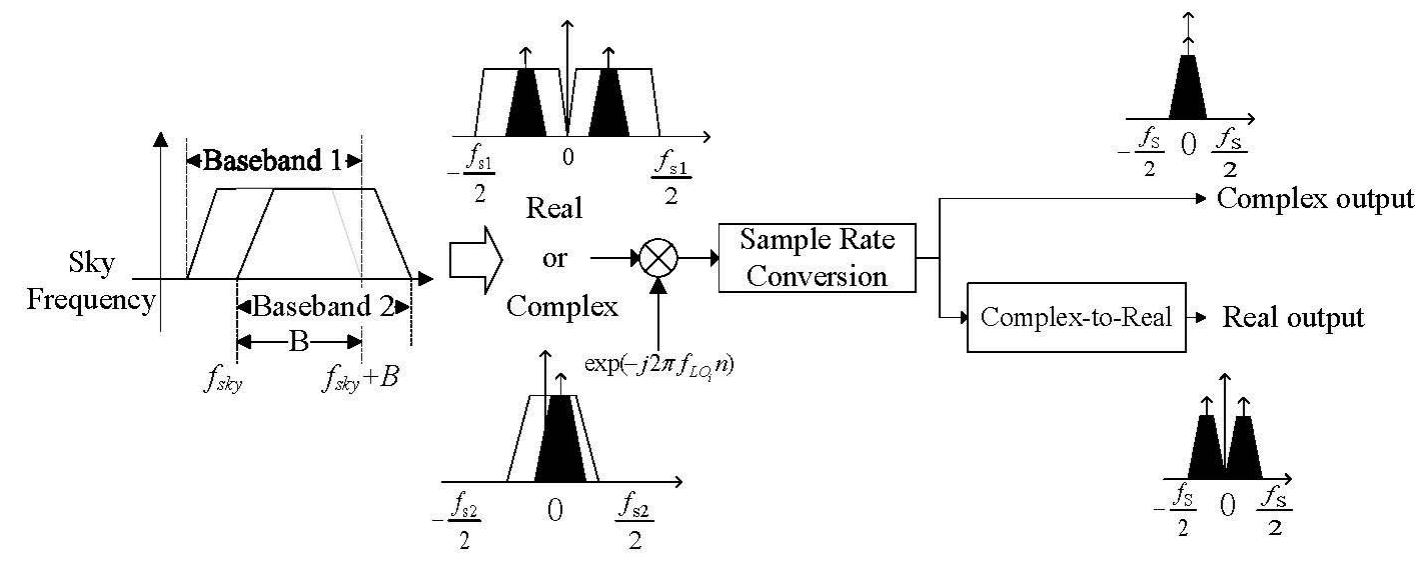

Fig. 1: Convert-before-correlation method.

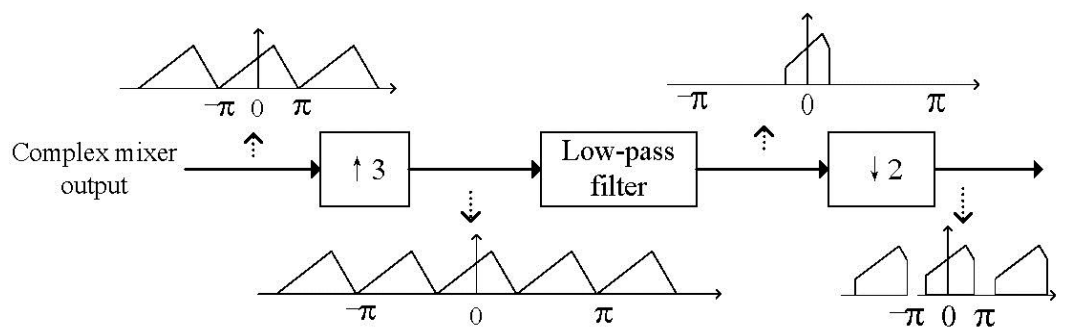

Fig. 2: Frequency domain effects of sample rate conversion.

Due to the ratio of sample rates could be rational number, the sample rate conversion should be designed cautiously to avoid aliasing. Assume the conversion ratio is $P / Q$, both $P$ and $Q$ are integer here. The sample rate is first increased by $P$, which is made by inserting $(P-1)$ zero between adjacent samples. Then a low-pass filter that stops at $B / 2 \mathrm{~Hz}$ is used for anti-aliasing. Then decimation is performed by selecting every $Q$ th point 
from the filtered data. Fig. 2 shows the demonstration of frequency domain effects when $P=3$ and $Q=2$. Polyphase filter banks could be used in filter design for computation reducing [8].

A computer simulation is made to verify our method. Real white Gauss noise data is generated at 8 Msps as immediate frequency (IF) signals, and heterogeneous data are selected with different bandwidth, sample rates and start IF frequencies. Independent white Gauss noise is added to baseband signals as noise. Signal to noise rates (SNR) of real and complex are both 10dB. Both real and complex data is transformed to real by data converters. The output is at $2 \mathrm{Msps}$ and the stop frequency of the low-pass filter is $0.99 \mathrm{MHz}$. Description of the subband selection and fringe phase are showed in Fig. 3.

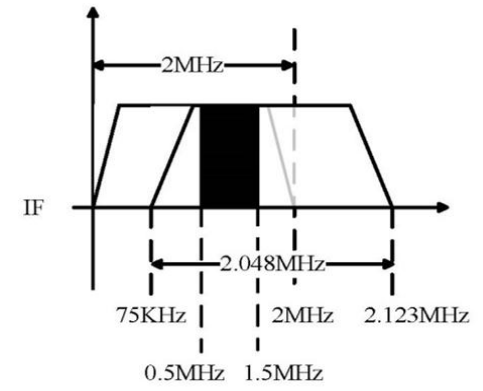

(a)

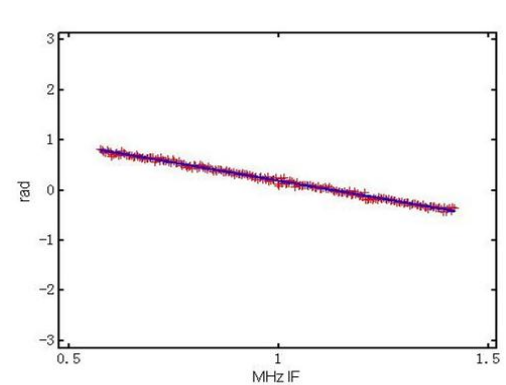

(b)

Fig. 3: (a) Subband selection. (b) Fringe phase results.

\section{The Direct FX Correlation Method for Heterogeneous Data}

Correlation between heterogeneous data could be performed directly in frequency domain by an improved FX correlator architecture. Data converters are not necessary so the computation is reduced. In normal FX correlator, data is first performed Fourier transform to get frequency domain representation and then the correlation is performed in frequency domain [2]. Integer samples delay compensation (ISDC) and fractional samples delay compensation (FSDC) eliminate the propagation delay with predict model. Fig. 4 shows the function graph of the direct FX correlator for a single baseline. The correlator is modified for heterogeneous data as followed. First, the DFT sizes for different DBEs could be different and arbitrary. Second, spectrum alignment module is designed to align the subband in DFT frequencies.

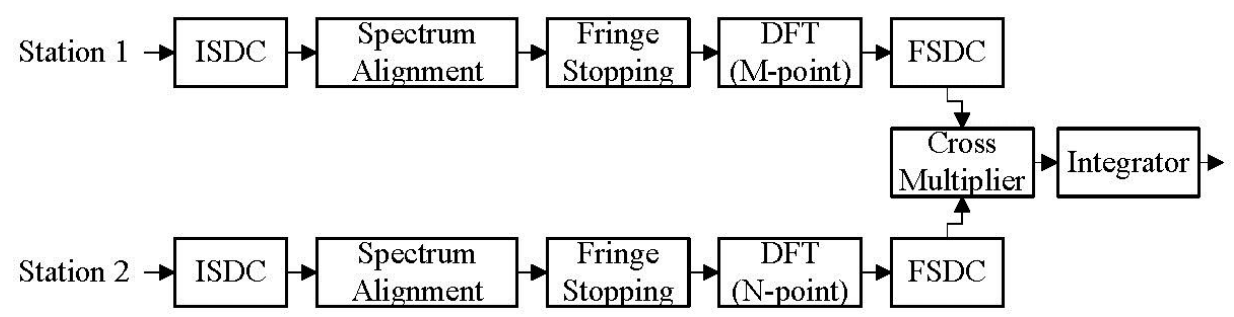

Fig. 4: Direct FX correlator.

The output of DFT is a data series versus discrete frequency. Spectrum alignment shifts the DFT frequencies of subbands to the same sky frequencies. The subband start frequency should be shifted to nearest DFT frequency term. Besides the spectrum alignment, the frequency resolution of heterogeneous data spectrum, which is the frequency value of the gap between adjacent DFT frequencies, should be the same. It is determined by sample rate and DFT size. It should be the equal to ensure valid correlation. Assume $f_{\text {real }}$ and $f_{\text {complex }}$ are the sample rates of heterogeneous data, $M$ and $N$ are the DFT sizes, then we have

$$
\frac{f_{\text {real }}}{M}=\frac{f_{\text {complex }}}{N} \text {. }
$$

DFT output with frequency included in the subband are multiplied after FSDC. Results are integrated and the propagation delay is obtained from the phases of cross-spectrum.

Here we use the real VLBI observation data from different type of DBEs to verify the direct FX correlation method. The observation for quasar source 1741-038 was performed by Chinese VLBI Network 
$(\mathrm{CVN})$. Signals coming were received in X-band and recorded by different DBEs, RSR and Mk-5B, in the same time. Station KM (located in Kunming) gave real data at $8 \mathrm{Msps}$ by Mk-5B, while station TM (located in Shanghai) used RSR to produce complex data at $4 \mathrm{Msps}{ }^{[9]}$. The IF frequency of heterogeneous data is $358.25 \mathrm{MHz}$. The baseline is about $1000 \mathrm{~km}$ length. The DFT sizes of RSR and Mk-5B are 256 and 512, so the frequency resolution is $15625 \mathrm{~Hz}$. As the start IF frequency of basebands are the same, frequency alignment is not required here. Although the entire $4 \mathrm{MHz}$ bandwidth could be used for correlation, the subband is chosen from $358.4 \mathrm{MHz}$ to $361.9 \mathrm{MHz}$ to verify our method. The fringes phases and fitting residuals are showed in Fig. 4.
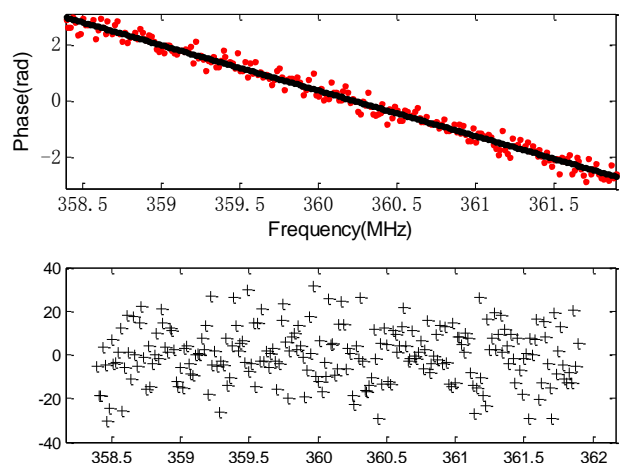

Fig. 4: Long baseline interference fringe phase using heterogeneous data.

\section{Conclusions}

This paper systematically discusses two methods to processing heterogeneous VLBI data. The convertbefore-correlation method use a convertor between DBEs and normal correlators, while direct FX correlator performs correlation directly for heterogenous data. Methods have been verified in computer simulations and quasar observations. In the further study, the direct FX correlator could be developed to have additional functions, such as fringe fitting.

\section{Acknowledgements}

We gratefully acknowledge support of the National Natural Science Foundation of China (U1531104) and NSF(11373061), Program of Shanghai Academic Research Leader (14XD1404300).

\section{References}

[1] A. R. Thompson, J. M. Moran, G. W. Swenson, Jr. Interferometry and synthesis in radio astronomy. John Wiley \& Sons, 2008.

[2] J. D. Romney. Theory of correlation in VLBI. Very Long Baseline Interferometry and the VLBA, 1995, 82: 17-37.

[3] A. Whitney. VLBI Standard Interface Specification. 2000 Annual Report, International VLBI Service for Geodesy and Astrometry. 2001, pp. 18-49.

[4] A. Whitney. The Mark 5B VLBI Data System. European VLBI Network on New Developments in VLBI Science and Technology, 2004, 1: 251-252.

[5] K. Takefuji, H. Takeuchi, M. Tsutsumi, et al. Next-generation A/D Sampler ADS3000+. VLBI2010 IVS 2010 General Meeting Proceedings. 2010, pp. 378-382.

[6] M. Mercolino, R. Maddè, L. Iess, et al. Results and future applications of the ESA Delta-DOR. ESA Bulletin,2006, 128: $68-74$

[7] H. Takeuchi, S. Horiuchi, C. Phillips, et al. VLBI tracking of the solar sail mission IKAROS. General Assembly and Scientific Symposium, 2011 XXXth URSI. IEEE. 2011, pp. 1-4.

[8] P. P. Vaidyanathan. Multirate digital filters, filter banks, polyphase networks, and applications: a tutorial. Proceedings of the IEEE. 1990, 78(1): 56-93.

[9] X. Li. The Design of Delta-DOR Common Receiver in Deep Space Instrumentation System (in Chinese), M.S., Southeast University, 2009. 